\title{
A Classical Cycle of Activity in the North Tropical Region of Jupiter, 1996-1999
}

\author{
John Rogers, Michael Foulkes, Hans-Joerg Mettig \\ British Astronomical Association, U.K.
}

\begin{abstract}
Jupiter's North Equatorial Belt (NEB) occasionally undergoes cycles of activity that consist of rapid broadening of the dark belt to the north, often accompanied by cyclonic bright "rift" activity, followed by development of large circulations in the expanded northern part (cyclonic dark "barges" and anticyclonic bright "portholes"), and a reddish colour across the belt (Rogers 1995). In 1996 there was a classic NEB broadening event with typical evolution over the following years. The broadening to the north began in 1996 April and spread around the belt by the summer. By 1997 April, there was an array of "barges" and "portholes" (white ovals) all around the broadened NEB, and increased redness of the belt. During 1997 the northern extension gradually reverted towards the normal bright albedo for this latitude, but the barges and ovals persisted. Indeed they still persist in 1999/00. Features in 1998/99/00 have included a rapidly-prograding white oval which destroyed barges ahead of it and had retrograding dark spots created following it; these retrograding spots have circulated as was observed in Voyager maps.
\end{abstract}

\section{References}

Rogers, J.H. 1995, The Giant Planet Jupiter (Cambridge: CUP) 